Acta Crystallographica Section E

Structure Reports

Online

ISSN 1600-5368

\section{5-Cyclohexyl-3-(3-fluorophenylsulfinyl)- 2-methyl-1-benzofuran}

\section{Hong Dae Choi, ${ }^{a}$ Pil Ja Seo ${ }^{a}$ and Uk Lee ${ }^{b *}$}

aDepartment of Chemistry, Dongeui University, San 24 Kaya-dong, Busanjin-gu, Busan 614-714, Republic of Korea, and ${ }^{\mathbf{b}}$ Department of Chemistry, Pukyong

National University, 599-1 Daeyeon 3-dong, Nam-gu, Busan 608-737, Republic of Korea

Correspondence e-mail: uklee@pknu.ac.kr

Received 23 May 2013; accepted 29 May 2013

Key indicators: single-crystal X-ray study; $T=173 \mathrm{~K}$; mean $\sigma(\mathrm{C}-\mathrm{C})=0.002 \AA$; $R$ factor $=0.042 ; w R$ factor $=0.124 ;$ data-to-parameter ratio $=19.2$.

In the title compound, $\mathrm{C}_{21} \mathrm{H}_{21} \mathrm{FO}_{2} \mathrm{~S}$, the cyclohexyl ring adopts a chair conformation. The 3-fluorophenyl ring makes a dihedral angle of $83.16(4)^{\circ}$ with the mean plane [r.m.s. deviation $=0.005$ (1) $\AA$ ] of the benzofuran ring system. In the crystal, molecules are linked by pairs of $\mathrm{C}-\mathrm{H} \cdot \cdots \pi$ interactions into inversion dimers, which are further packed into stacks along the $a$-axis direction by $\mathrm{C}-\mathrm{H} \cdots \pi$ interactions.

\section{Related literature}

For background information and the crystal structures of related compounds, see: Choi et al. (2011, 2012a,b).

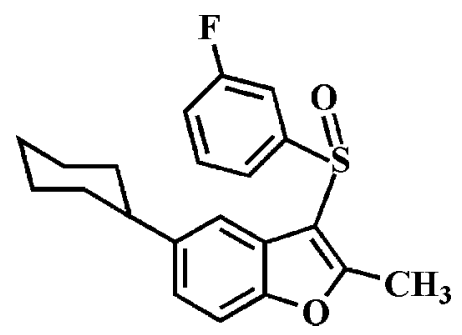

\section{Experimental}

Crystal data

$\mathrm{C}_{21} \mathrm{H}_{21} \mathrm{FO}_{2} \mathrm{~S}$

$M_{r}=356.44$

Triclinic, $P \overline{1}$

$a=8.9147$ (1) ̊ $\gamma=97.439$ (1)

$V=880.44(3) \AA^{3}$

$Z=2$

Mo $K \alpha$ radiation

Data collection

Bruker SMART APEXII CCD diffractometer

Absorption correction: multi-scan (SADABS; Bruker, 2009)

$T_{\min }=0.692, T_{\max }=0.746$

Refinement

$R\left[F^{2}>2 \sigma\left(F^{2}\right)\right]=0.042$

$w R\left(F^{2}\right)=0.124$

$S=1.04$

4369 reflections $\mu=0.21 \mathrm{~mm}^{-1}$

$T=173 \mathrm{~K}$

$0.33 \times 0.31 \times 0.29 \mathrm{~mm}$

19435 measured reflections 4369 independent reflections 3897 reflections with $I>2 \sigma(I)$ $R_{\text {int }}=0.025$

227 parameters

$\mathrm{H}$-atom parameters constrained

$\Delta \rho_{\max }=0.94{\mathrm{e} \AA^{-3}}^{-3}$

$\Delta \rho_{\min }=-0.41{\mathrm{e} \AA^{-3}}^{-3}$

Table 1

Hydrogen-bond geometry $\left(\AA,{ }^{\circ}\right)$.

$C g 1$ and $C g 2$ are the centroids of the $\mathrm{C} 1 / \mathrm{C} 2 / \mathrm{C} 7 / \mathrm{O} 1 / \mathrm{C} 8$ furan ring and the $\mathrm{C} 2-$ C7 benzene ring, respectively.

\begin{tabular}{lllll}
\hline$D-\mathrm{H} \cdots A$ & $D-\mathrm{H}$ & $\mathrm{H} \cdots A$ & $D \cdots A$ & $D-\mathrm{H} \cdots A$ \\
\hline $\mathrm{C} 13-\mathrm{H} 13 A \cdots C g 1^{\mathrm{i}}$ & 0.99 & 3.00 & $3.697(1)$ & 128 \\
$\mathrm{C} 14-\mathrm{H} 14 B \cdots C g 2^{\mathrm{i}}$ & 0.99 & 2.91 & $3.569(1)$ & 125 \\
$\mathrm{C} 19-\mathrm{H} 19 \cdots C g 2^{\mathrm{ii}}$ & 0.95 & 2.90 & $3.677(1)$ & 140 \\
\hline
\end{tabular}

Symmetry codes: (i) $-x+1,-y,-z+1$; (ii) $x+1, y, z$.

Data collection: APEX2 (Bruker, 2009); cell refinement: SAINT (Bruker, 2009); data reduction: $S A I N T$; $\operatorname{program}(\mathrm{s})$ used to solve structure: SHELXS97 (Sheldrick, 2008); program(s) used to refine structure: SHELXL97 (Sheldrick, 2008); molecular graphics: ORTEP-3 for Windows (Farrugia, 2012) and DIAMOND (Brandenburg, 1998); software used to prepare material for publication: SHELXL97.

This work was supported by the Blue-Bio Industry Regional Innovation Center (RIC08-06-07) at Dongeui University as an RIC program under the Ministry of Knowledge Economy and Busan city.

Supplementary data and figures for this paper are available from the IUCr electronic archives (Reference: BX2442).

\title{
References
}

Brandenburg, K. (1998). DIAMOND. Crystal Impact GbR, Bonn, Germany. Bruker (2009). APEX2, SADABS and SAINT. Bruker AXS Inc., Madison, Wisconsin, USA.

Choi, H. D., Seo, P. J. \& Lee, U. (2012a). Acta Cryst. E68, o205.

Choi, H. D., Seo, P. J. \& Lee, U. (2012b). Acta Cryst. E68, o947.

Choi, H. D., Seo, P. J., Son, B. W. \& Lee, U. (2011). Acta Cryst. E67, o1157.

Farrugia, L. J. (2012). J. Appl. Cryst. 45, 849-854.

Sheldrick, G. M. (2008). Acta Cryst. A64, 112-122. 


\section{supporting information}

Acta Cryst. (2013). E69, o1026 [https://doi.org/10.1107/S160053681301475X]

\section{5-Cyclohexyl-3-(3-fluorophenylsulfinyl)-2-methyl-1-benzofuran}

\section{Hong Dae Choi, Pil Ja Seo and Uk Lee}

\section{S1. Comment}

As a part of our continuing study of 5-cyclohexyl-2-methyl-1-benzofuran derivatives containing phenylsulfinyl (Choi et al., 2011), 4-bromophenylsulfinyl (Choi et al., 2012a) and 4-methylphenylsulfinyl (Choi et al., 2012b)substituents in 3position, we report herein the crystal structure of the title compound.

In the title molecule (Fig. 1), the benzofuran unit is essentially planar, with a mean deviation of 0.005 (1) $\AA$ from the least-squares plane defined by the nine constituent atoms. The cyclohexyl ring has the chair form. The dihedral angle between the 3-fluorophenyl ring and the mean plane of the benzofuran ring system is $83.16(4)^{\circ}$. In the crystal structure (Fig. 2), molecules are connected by pairs of $\mathrm{C}-\mathrm{H}^{\cdots} \cdots \pi$ interactions into dimers, which are further packed into stacks along the a axis by $\mathrm{C}-\mathrm{H}^{\cdots} \cdots \pi$ interactions (Table $1, \mathrm{Cg} 1$ and $\mathrm{Cg} 2$ are the centroids of the $\mathrm{C} 1 / \mathrm{C} 2 / \mathrm{C} 7 / \mathrm{O} 1 / \mathrm{C} 8$ furan ring and the $\mathrm{C} 2-\mathrm{C} 7$ benzene ring, respectively).

\section{S2. Experimental}

3-Chloroperoxybenzoic acid $(77 \%, 202 \mathrm{mg}, 0.9 \mathrm{mmol})$ was added in small portions to a stirred solution of 5-cyclohexyl-3-(3-fluorophenylsulfanyl)-2-methyl-1-benzofuran $(272 \mathrm{mg}, 0.9 \mathrm{mmol})$ in dichloromethane $(30 \mathrm{~mL})$ at $273 \mathrm{~K}$. After being stirred at room temperature for $4 \mathrm{~h}$, the mixture was washed with saturated sodium bicarbonate solution and the organic layer was separated, dried over magnesium sulfate, filtered and concentrated at reduced pressure. The residue was purified by column chromatography (hexane-ethyl acetate, $4: 1 \mathrm{v} / \mathrm{v}$ ) to afford the title compound as a colorless solid [yield 68\%, m.p. 403-404 K; $R_{\mathrm{f}}=0.43$ (hexane-ethyl acetate, 4:1 v/v)]. Single crystals suitable for X-ray diffraction were prepared by slow evaporation of a solution of the title compound in ethyl acetate at room temperature.

\section{S3. Refinement}

All $\mathrm{H}$ atoms were positioned geometrically and refined using a riding model, with $\mathrm{C}-\mathrm{H}=0.95 \AA$ for aryl, $1.00 \AA$ for methine, $0.99 \AA$ for methylene and $0.98 \AA$ for methyl $\mathrm{H}$ atoms, respectively. $U_{\mathrm{iso}}(\mathrm{H})=1.2 U_{\mathrm{eq}}(\mathrm{C})$ for aryl, methine and methylene, and $1.5 U_{\mathrm{eq}}(\mathrm{C})$ for methyl $\mathrm{H}$ atoms. The positions of methyl hydrogens were optimized rotationally. 


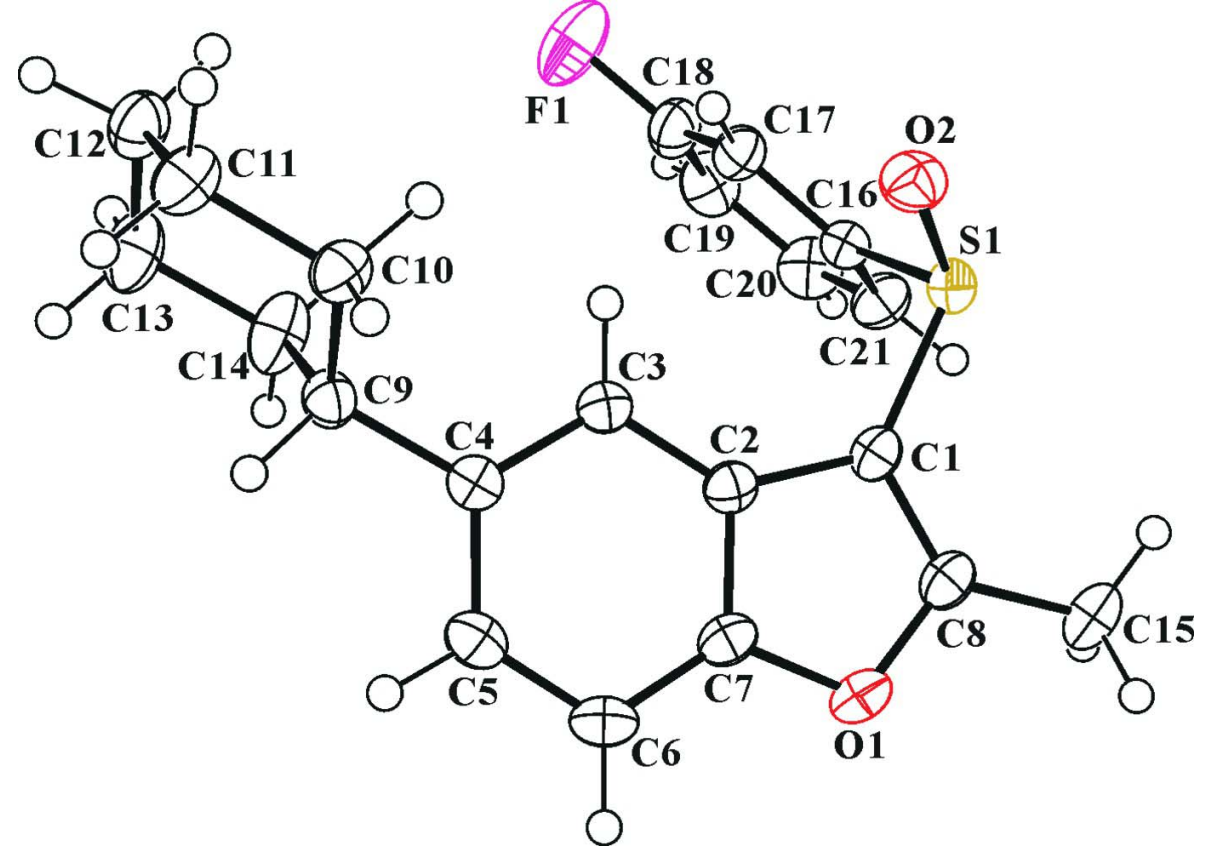

Figure 1

The molecular structure of the title compound with the atom numbering scheme. Displacement ellipsoids are drawn at the $50 \%$ probability level. $\mathrm{H}$ atoms are presented as small spheres of arbitrary radius.

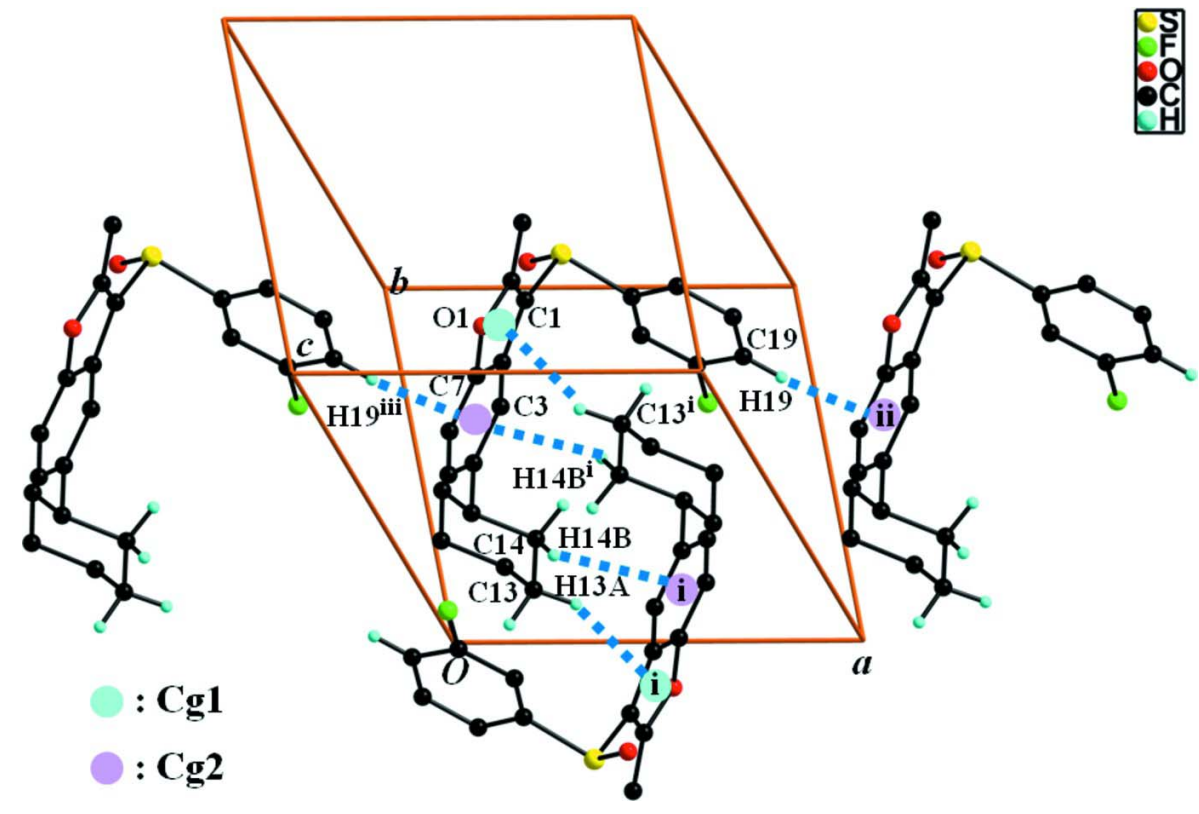

Figure 2

A view of the $\mathrm{C}-\mathrm{H} \cdots \pi$ interactions (dotted lines) in the crystal structure of the title compound. $\mathrm{H}$ atoms non-participating in hydrogen-bonding were omitted for clarity. [Symmetry codes: (i) $-x+1,-y,-z+1$; (ii) $x+1, y, z$; (iii) $x-1, y, z$.] 


\section{5-Cyclohexyl-3-(3-fluorophenylsulfinyl)-2-methyl-1-benzofuran}

Crystal data

$\mathrm{C}_{21} \mathrm{H}_{21} \mathrm{FO}_{2} \mathrm{~S}$

$M_{r}=356.44$

Triclinic, $P \overline{1}$

Hall symbol: -P 1

$a=8.9147(1) \AA$

$b=10.1270(2) \AA$

$c=10.5101(2) \AA$

$\alpha=90.376(1)^{\circ}$

$\beta=110.407(1)^{\circ}$

$\gamma=97.439(1)^{\circ}$

$V=880.44(3) \AA^{3}$

\section{Data collection}

Bruker SMART APEXII CCD diffractometer

Radiation source: rotating anode

Graphite multilayer monochromator

Detector resolution: 10.0 pixels $\mathrm{mm}^{-1}$

$\varphi$ and $\omega$ scans

Absorption correction: multi-scan

(SADABS; Bruker, 2009)

$T_{\min }=0.692, T_{\max }=0.746$

\section{Refinement}

Refinement on $F^{2}$

Least-squares matrix: full

$R\left[F^{2}>2 \sigma\left(F^{2}\right)\right]=0.042$

$w R\left(F^{2}\right)=0.124$

$S=1.04$

4369 reflections

227 parameters

0 restraints

Primary atom site location: structure-invariant direct methods
$Z=2$

$F(000)=376$

$D_{\mathrm{x}}=1.345 \mathrm{Mg} \mathrm{m}^{-3}$

Melting point $=403-404 \mathrm{~K}$

Mo $K \alpha$ radiation, $\lambda=0.71073 \AA$

Cell parameters from 9475 reflections

$\theta=2.6-28.3^{\circ}$

$\mu=0.21 \mathrm{~mm}^{-1}$

$T=173 \mathrm{~K}$

Block, colourless

$0.33 \times 0.31 \times 0.29 \mathrm{~mm}$

19435 measured reflections

4369 independent reflections

3897 reflections with $I>2 \sigma(I)$

$R_{\text {int }}=0.025$

$\theta_{\max }=28.3^{\circ}, \theta_{\min }=2.0^{\circ}$

$h=-11 \rightarrow 11$

$k=-13 \rightarrow 13$

$l=-13 \rightarrow 14$

\section{Special details}

Secondary atom site location: difference Fourier map

Hydrogen site location: difference Fourier map

$\mathrm{H}$-atom parameters constrained

$w=1 /\left[\sigma^{2}\left(F_{\mathrm{o}}^{2}\right)+(0.0748 P)^{2}+0.2924 P\right]$

where $P=\left(F_{\mathrm{o}}^{2}+2 F_{\mathrm{c}}^{2}\right) / 3$

$(\Delta / \sigma)_{\max }<0.001$

$\Delta \rho_{\max }=0.94 \mathrm{e} \AA^{-3}$

$\Delta \rho_{\min }=-0.41 \mathrm{e} \AA^{-3}$

Geometry. All esds (except the esd in the dihedral angle between two 1.s. planes) are estimated using the full covariance matrix. The cell esds are taken into account individually in the estimation of esds in distances, angles and torsion angles; correlations between esds in cell parameters are only used when they are defined by crystal symmetry. An approximate (isotropic) treatment of cell esds is used for estimating esds involving l.s. planes.

Refinement. Refinement of $\mathrm{F}^{2}$ against ALL reflections. The weighted R-factor $\mathrm{wR}$ and goodness of fit $\mathrm{S}$ are based on $\mathrm{F}^{2}$, conventional $R$-factors $R$ are based on $F$, with $F$ set to zero for negative $F^{2}$. The threshold expression of $F^{2}>2 \operatorname{sigma}\left(\mathrm{F}^{2}\right)$ is used only for calculating R-factors(gt) etc. and is not relevant to the choice of reflections for refinement. R-factors based on $\mathrm{F}^{2}$ are statistically about twice as large as those based on F, and R- factors based on ALL data will be even larger.

Fractional atomic coordinates and isotropic or equivalent isotropic displacement parameters $\left(\AA^{2}\right)$

\begin{tabular}{lllll}
\hline & $x$ & $y$ & $z$ & $U_{\text {iso }} / U_{\text {eq }}$ \\
\hline S1 & $0.65884(4)$ & $0.48559(3)$ & $0.79845(3)$ & $0.02628(11)$ \\
F1 & $0.84222(15)$ & $0.35909(14)$ & $0.41074(11)$ & $0.0615(4)$ \\
O1 & $0.47089(13)$ & $0.17436(11)$ & $0.94217(10)$ & $0.0312(2)$
\end{tabular}




\begin{tabular}{|c|c|c|c|c|}
\hline $\mathrm{O} 2$ & $0.54304(14)$ & $0.54929(11)$ & $0.68662(11)$ & $0.0358(3)$ \\
\hline $\mathrm{C} 1$ & $0.55705(16)$ & $0.33517(14)$ & $0.82691(13)$ & $0.0252(3)$ \\
\hline $\mathrm{C} 2$ & $0.44498(15)$ & $0.23532(13)$ & $0.72755(13)$ & $0.0235(3)$ \\
\hline $\mathrm{C} 3$ & $0.38240(16)$ & $0.21856(13)$ & $0.58605(13)$ & $0.0242(3)$ \\
\hline $\mathrm{H} 3$ & 0.4136 & 0.2834 & 0.5317 & $0.029^{*}$ \\
\hline $\mathrm{C} 4$ & $0.27338(16)$ & $0.10501(13)$ & $0.52593(13)$ & $0.0248(3)$ \\
\hline $\mathrm{C} 5$ & $0.22910(18)$ & $0.01022(15)$ & $0.60832(15)$ & $0.0307(3)$ \\
\hline H5 & 0.1549 & -0.0669 & 0.5659 & $0.037 *$ \\
\hline C6 & $0.28993(18)$ & $0.02523(16)$ & $0.74949(15)$ & $0.0323(3)$ \\
\hline H6 & 0.2597 & -0.0393 & 0.8045 & $0.039 *$ \\
\hline $\mathrm{C} 7$ & $0.39658(16)$ & $0.13922(14)$ & $0.80477(13)$ & $0.0268(3)$ \\
\hline $\mathrm{C} 8$ & $0.56642(17)$ & $0.29422(15)$ & $0.95219(14)$ & $0.0281(3)$ \\
\hline C9 & $0.20412(16)$ & $0.08110(14)$ & $0.37274(14)$ & $0.0264(3)$ \\
\hline H9 & 0.1189 & 0.0013 & 0.3508 & $0.032 *$ \\
\hline $\mathrm{C} 10$ & $0.12469(18)$ & $0.19886(15)$ & $0.30242(14)$ & $0.0292(3)$ \\
\hline H10A & 0.0361 & 0.2136 & 0.3349 & $0.035^{*}$ \\
\hline H10B & 0.2054 & 0.2804 & 0.3272 & $0.035^{*}$ \\
\hline C11 & $0.05690(19)$ & $0.17497(17)$ & $0.14790(15)$ & $0.0341(3)$ \\
\hline H11A & -0.0336 & 0.1006 & 0.1225 & $0.041^{*}$ \\
\hline H11B & 0.0138 & 0.2558 & 0.1061 & $0.041^{*}$ \\
\hline $\mathrm{C} 12$ & $0.18461(19)$ & $0.14180(17)$ & $0.09251(15)$ & $0.0350(3)$ \\
\hline $\mathrm{H} 12 \mathrm{~A}$ & 0.1342 & 0.1200 & -0.0066 & $0.042 *$ \\
\hline H12B & 0.2683 & 0.2205 & 0.1072 & $0.042^{*}$ \\
\hline $\mathrm{C} 13$ & $0.2628(2)$ & 0.02487 (19) & $0.16166(16)$ & $0.0408(4)$ \\
\hline H13A & 0.3497 & 0.0087 & 0.1277 & $0.049^{*}$ \\
\hline H13B & 0.1812 & -0.0561 & 0.1386 & $0.049^{*}$ \\
\hline C14 & $0.3336(2)$ & 0.05058 (19) & $0.31578(16)$ & $0.0396(4)$ \\
\hline H14A & 0.4218 & 0.1269 & 0.3393 & $0.048^{*}$ \\
\hline H14B & 0.3803 & -0.0288 & 0.3581 & $0.048^{*}$ \\
\hline $\mathrm{C} 15$ & $0.6580(2)$ & $0.35137(18)$ & $1.09261(15)$ & $0.0377(4)$ \\
\hline H15A & 0.6953 & 0.4463 & 1.0897 & $0.057^{*}$ \\
\hline H15B & 0.7515 & 0.3045 & 1.1339 & $0.057^{*}$ \\
\hline $\mathrm{H} 15 \mathrm{C}$ & 0.5878 & 0.3410 & 1.1468 & $0.057^{*}$ \\
\hline $\mathrm{C} 16$ & $0.79260(16)$ & $0.41782(13)$ & $0.72905(13)$ & $0.0248(3)$ \\
\hline C17 & 0.75975 (17) & $0.41440(15)$ & $0.59050(14)$ & $0.0291(3)$ \\
\hline H17 & 0.6656 & 0.4446 & 0.5295 & $0.035^{*}$ \\
\hline $\mathrm{C} 18$ & $0.8701(2)$ & $0.36513(17)$ & $0.54482(16)$ & $0.0354(3)$ \\
\hline C19 & $1.0094(2)$ & $0.32320(17)$ & $0.62948(18)$ & $0.0398(4)$ \\
\hline H19 & 1.0829 & 0.2907 & 0.5939 & $0.048^{*}$ \\
\hline $\mathrm{C} 20$ & $1.0398(2)$ & $0.32951(18)$ & $0.76767(18)$ & $0.0404(4)$ \\
\hline $\mathrm{H} 20$ & 1.1352 & 0.3006 & 0.8281 & $0.049^{*}$ \\
\hline $\mathrm{C} 21$ & $0.93245(18)$ & $0.37764(17)$ & $0.81917(15)$ & $0.0344(3)$ \\
\hline $\mathrm{H} 21$ & 0.9542 & 0.3830 & 0.9143 & $0.041^{*}$ \\
\hline
\end{tabular}

Atomic displacement parameters $\left(\AA^{2}\right)$

\begin{tabular}{lllllll}
\hline & $U^{11}$ & $U^{22}$ & $U^{33}$ & $U^{12}$ & $U^{13}$ & $U^{23}$ \\
\hline S1 & $0.02997(19)$ & $0.02451(18)$ & $0.02462(17)$ & $0.00348(13)$ & $0.01014(13)$ & $-0.00131(12)$
\end{tabular}




\begin{tabular}{|c|c|c|c|c|c|c|}
\hline $\mathrm{F} 1$ & $0.0640(7)$ & $0.0929(10)$ & $0.0356(5)$ & $0.0216(7)$ & $0.0239(5)$ & $-0.0039(6)$ \\
\hline O1 & $0.0346(5)$ & $0.0388(6)$ & $0.0242(5)$ & $0.0057(4)$ & $0.0152(4)$ & $0.0056(4)$ \\
\hline $\mathrm{O} 2$ & $0.0386(6)$ & $0.0332(6)$ & $0.0378(6)$ & $0.0126(5)$ & $0.0136(5)$ & 0.0070 \\
\hline $\mathrm{C} 1$ & $0.0261(6)$ & $0.0285(6)$ & $0.0226(6)$ & $0.0049(5)$ & $0.0103(5)$ & $0.0001(5)$ \\
\hline $\mathrm{C} 2$ & $0.0227(6)$ & $0.0254(6)$ & $0.0248(6)$ & $0.0054(5)$ & $0.0109(5)$ & $0.0021(5)$ \\
\hline C3 & $0.0245(6)$ & $0.0247(6)$ & $0.0244(6)$ & $0.0038(5)$ & $0.0097(5)$ & $0.0029(5)$ \\
\hline $\mathrm{C} 4$ & 0.0230 & $0.0247(6)$ & $0.0271(6)$ & $0.0042(5)$ & $0.0091(5)$ & $0.0019(5)$ \\
\hline $\mathrm{C} 5$ & $0.0284(7)$ & $0.0274(7)$ & $0.0357(7)$ & $-0.0009(5)$ & $0.0125(6)$ & $0.0020(6)$ \\
\hline C6 & $0.0331(7)$ & $0.0321(7)$ & $0.0355(7)$ & $0.0014(6)$ & $0.0177(6)$ & 0.0089 (6) \\
\hline $\mathrm{C} 7$ & $0.0269(6)$ & $0.0319(7)$ & $0.0252(6)$ & $0.0058(5)$ & $0.0132(5)$ & $0.0042(5)$ \\
\hline $\mathrm{C} 8$ & $0.0287(6)$ & $0.0343(7)$ & $0.0245(6)$ & $0.0079(6)$ & $0.0121(5)$ & $0.0012(5)$ \\
\hline C9 & $0.0254(6)$ & $0.0253(6)$ & $0.0261(6)$ & $0.0021(5)$ & $0.0067(5)$ & $-0.0004(5)$ \\
\hline $\mathrm{C} 10$ & $0.0319(7)$ & $0.0306(7)$ & $0.0275(6)$ & 0.0093 (6) & $0.0118(5)$ & $0.0020(5)$ \\
\hline $\mathrm{C} 11$ & $0.0338(7)$ & $0.0420(8)$ & $0.0276(7)$ & $0.0119(6)$ & $0.0099(6)$ & 0.0039 (6) \\
\hline $\mathrm{C} 12$ & $0.0371(8)$ & $0.0417(8)$ & $0.0284(7)$ & $0.0035(6)$ & $0.0149(6)$ & $-0.0027(6)$ \\
\hline $\mathrm{C} 13$ & $0.0418(8)$ & $0.0500(10)$ & $0.0311(7)$ & $0.0180(7)$ & $0.0095(6)$ & $-0.0080(7)$ \\
\hline $\mathrm{C} 14$ & $0.0349(8)$ & $0.0532(10)$ & $0.0305(7)$ & $0.0205(7)$ & $0.0063(6)$ & $-0.0063(7)$ \\
\hline $\mathrm{C} 15$ & $0.0426(8)$ & $0.0493(9)$ & $0.0229(6)$ & $0.0084(7)$ & $0.0130(6)$ & $-0.0009(6)$ \\
\hline $\mathrm{C} 16$ & $0.0260(6)$ & $0.0229(6)$ & 0.0255 & $0.0021(5)$ & $0.0096(5)$ & $0.0016(5)$ \\
\hline $\mathrm{C} 17$ & $0.0283(7)$ & $0.0322(7)$ & $0.0252(6)$ & 0.0040 & $0.0076(5)$ & $0.0013(5)$ \\
\hline $\mathrm{C} 18$ & $0.0383(8)$ & $0.0393(8)$ & $0.0308(7)$ & $0.0021(6)$ & $0.0162(6)$ & -0.0039 (6) \\
\hline C19 & $0.0343(8)$ & $0.0400(9)$ & $0.0499(9)$ & $0.0083(7)$ & $0.0199(7)$ & $-0.0028(7)$ \\
\hline $\mathrm{C} 20$ & $0.0310(8)$ & $0.0447(9)$ & $0.0449(9)$ & $0.0129(7)$ & $0.0098(7)$ & $0.0071(7)$ \\
\hline $\mathrm{C} 21$ & $0.0328(7)$ & $0.0406(8)$ & $0.0278(7)$ & $0.0089(6)$ & $0.0069(6)$ & $0.0061(6)$ \\
\hline
\end{tabular}

Geometric parameters ( $\AA, \stackrel{\circ}{)})$

\begin{tabular}{llll}
\hline $\mathrm{S} 1-\mathrm{O} 2$ & $1.4831(11)$ & $\mathrm{C} 11-\mathrm{C} 12$ & $1.519(2)$ \\
$\mathrm{S} 1-\mathrm{C} 1$ & $1.7523(15)$ & $\mathrm{C} 11-\mathrm{H} 11 \mathrm{~A}$ & 0.9900 \\
$\mathrm{~S} 1-\mathrm{C} 16$ & $1.7992(14)$ & $\mathrm{C} 11-\mathrm{H} 11 \mathrm{~B}$ & 0.9900 \\
$\mathrm{~F} 1-\mathrm{C} 18$ & $1.3424(18)$ & $\mathrm{C} 12-\mathrm{C} 13$ & 0.9900 \\
$\mathrm{O} 1-\mathrm{C} 8$ & $1.3703(18)$ & $\mathrm{C} 12-\mathrm{H} 12 \mathrm{~A}$ & 0.9900 \\
$\mathrm{O} 1-\mathrm{C} 7$ & $1.3842(16)$ & $\mathrm{C} 12-\mathrm{H} 12 \mathrm{~B}$ & $1.526(2)$ \\
$\mathrm{C} 1-\mathrm{C} 8$ & $1.3609(19)$ & $\mathrm{C} 13-\mathrm{C} 14$ & 0.9900 \\
$\mathrm{C} 1-\mathrm{C} 2$ & $1.4520(18)$ & $\mathrm{C} 13-\mathrm{H} 13 \mathrm{~A}$ & 0.9900 \\
$\mathrm{C} 2-\mathrm{C} 7$ & $1.3920(19)$ & $\mathrm{C} 13-\mathrm{H} 13 \mathrm{~B}$ & 0.9900 \\
$\mathrm{C} 2-\mathrm{C} 3$ & $1.3948(17)$ & $\mathrm{C} 14-\mathrm{H} 14 \mathrm{~A}$ & 0.9900 \\
$\mathrm{C} 3-\mathrm{C} 4$ & $1.3925(19)$ & $\mathrm{C} 14-\mathrm{H} 14 \mathrm{~B}$ & 0.9800 \\
$\mathrm{C} 3-\mathrm{H} 3$ & $\mathrm{C} 15-\mathrm{H} 15 \mathrm{~A}$ & 0.9800 \\
$\mathrm{C} 4-\mathrm{C} 5$ & $\mathrm{C} 15-\mathrm{H} 15 \mathrm{~B}$ & 0.9800 \\
$\mathrm{C} 4-\mathrm{C} 9$ & $1.406(2)$ & $\mathrm{C} 15-\mathrm{H} 15 \mathrm{C}$ & $1.3809(18)$ \\
$\mathrm{C} 5-\mathrm{C} 6$ & $1.5149(18)$ & $\mathrm{C} 16-\mathrm{C} 17$ & $1.3891(19)$ \\
$\mathrm{C} 5-\mathrm{H} 5$ & $1.390(2)$ & $\mathrm{C} 16-\mathrm{C} 21$ & $1.379(2)$ \\
$\mathrm{C} 6-\mathrm{C} 7$ & 0.9500 & $\mathrm{C} 17-\mathrm{C} 18$ & 0.9500 \\
$\mathrm{C} 6-\mathrm{H} 6$ & $1.377(2)$ & $\mathrm{C} 17-\mathrm{H} 17$ & $1.373(2)$ \\
$\mathrm{C} 8-\mathrm{C} 15$ & 0.9500 & $\mathrm{C} 18-\mathrm{C} 19$ & $1.381(2)$ \\
$\mathrm{C} 9-\mathrm{C} 10$ & $1.4852(19)$ & $\mathrm{C} 19-\mathrm{C} 20$ & 0.9500 \\
$\mathrm{C} 9-\mathrm{C} 14$ & $1.5281(19)$ & $\mathrm{C} 19-\mathrm{H} 19$ & \\
& $1.536(2)$ & & \\
& & &
\end{tabular}




\begin{tabular}{|c|c|c|c|}
\hline $\mathrm{C} 9-\mathrm{H} 9$ & 1.0000 & $\mathrm{C} 20-\mathrm{C} 21$ & $1.387(2)$ \\
\hline $\mathrm{C} 10-\mathrm{C} 11$ & $1.5277(19)$ & $\mathrm{C} 20-\mathrm{H} 20$ & 0.9500 \\
\hline $\mathrm{C} 10-\mathrm{H} 10 \mathrm{~A}$ & 0.9900 & $\mathrm{C} 21-\mathrm{H} 21$ & 0.9500 \\
\hline $\mathrm{C} 10-\mathrm{H} 10 \mathrm{~B}$ & 0.9900 & & \\
\hline $\mathrm{O} 2-\mathrm{S} 1-\mathrm{C} 1$ & $107.99(7)$ & $\mathrm{C} 10-\mathrm{C} 11-\mathrm{H} 11 \mathrm{~B}$ & 109.2 \\
\hline $\mathrm{O} 2-\mathrm{S} 1-\mathrm{C} 16$ & $107.08(6)$ & $\mathrm{H} 11 \mathrm{~A}-\mathrm{C} 11-\mathrm{H} 11 \mathrm{~B}$ & 107.9 \\
\hline $\mathrm{C} 1-\mathrm{S} 1-\mathrm{C} 16$ & $98.41(6)$ & $\mathrm{C} 13-\mathrm{C} 12-\mathrm{C} 11$ & $110.99(13)$ \\
\hline $\mathrm{C} 8-\mathrm{O} 1-\mathrm{C} 7$ & $106.54(11)$ & $\mathrm{C} 13-\mathrm{C} 12-\mathrm{H} 12 \mathrm{~A}$ & 109.4 \\
\hline $\mathrm{C} 8-\mathrm{C} 1-\mathrm{C} 2$ & $107.28(12)$ & $\mathrm{C} 11-\mathrm{C} 12-\mathrm{H} 12 \mathrm{~A}$ & 109.4 \\
\hline $\mathrm{C} 8-\mathrm{C} 1-\mathrm{S} 1$ & $124.17(11)$ & $\mathrm{C} 13-\mathrm{C} 12-\mathrm{H} 12 \mathrm{~B}$ & 109.4 \\
\hline $\mathrm{C} 2-\mathrm{C} 1-\mathrm{S} 1$ & $128.51(10)$ & $\mathrm{C} 11-\mathrm{C} 12-\mathrm{H} 12 \mathrm{~B}$ & 109.4 \\
\hline $\mathrm{C} 7-\mathrm{C} 2-\mathrm{C} 3$ & $119.47(12)$ & $\mathrm{H} 12 \mathrm{~A}-\mathrm{C} 12-\mathrm{H} 12 \mathrm{~B}$ & 108.0 \\
\hline $\mathrm{C} 7-\mathrm{C} 2-\mathrm{C} 1$ & $104.60(11)$ & $\mathrm{C} 12-\mathrm{C} 13-\mathrm{C} 14$ & $111.35(13)$ \\
\hline $\mathrm{C} 3-\mathrm{C} 2-\mathrm{C} 1$ & $135.92(13)$ & $\mathrm{C} 12-\mathrm{C} 13-\mathrm{H} 13 \mathrm{~A}$ & 109.4 \\
\hline $\mathrm{C} 4-\mathrm{C} 3-\mathrm{C} 2$ & $118.74(12)$ & $\mathrm{C} 14-\mathrm{C} 13-\mathrm{H} 13 \mathrm{~A}$ & 109.4 \\
\hline $\mathrm{C} 4-\mathrm{C} 3-\mathrm{H} 3$ & 120.6 & $\mathrm{C} 12-\mathrm{C} 13-\mathrm{H} 13 \mathrm{~B}$ & 109.4 \\
\hline $\mathrm{C} 2-\mathrm{C} 3-\mathrm{H} 3$ & 120.6 & $\mathrm{C} 14-\mathrm{C} 13-\mathrm{H} 13 \mathrm{~B}$ & 109.4 \\
\hline $\mathrm{C} 3-\mathrm{C} 4-\mathrm{C} 5$ & $119.68(13)$ & $\mathrm{H} 13 \mathrm{~A}-\mathrm{C} 13-\mathrm{H} 13 \mathrm{~B}$ & 108.0 \\
\hline $\mathrm{C} 3-\mathrm{C} 4-\mathrm{C} 9$ & $120.75(12)$ & $\mathrm{C} 13-\mathrm{C} 14-\mathrm{C} 9$ & $111.27(12)$ \\
\hline $\mathrm{C} 5-\mathrm{C} 4-\mathrm{C} 9$ & $119.56(12)$ & $\mathrm{C} 13-\mathrm{C} 14-\mathrm{H} 14 \mathrm{~A}$ & 109.4 \\
\hline $\mathrm{C} 6-\mathrm{C} 5-\mathrm{C} 4$ & $122.44(13)$ & $\mathrm{C} 9-\mathrm{C} 14-\mathrm{H} 14 \mathrm{~A}$ & 109.4 \\
\hline $\mathrm{C} 6-\mathrm{C} 5-\mathrm{H} 5$ & 118.8 & $\mathrm{C} 13-\mathrm{C} 14-\mathrm{H} 14 \mathrm{~B}$ & 109.4 \\
\hline $\mathrm{C} 4-\mathrm{C} 5-\mathrm{H} 5$ & 118.8 & $\mathrm{C} 9-\mathrm{C} 14-\mathrm{H} 14 \mathrm{~B}$ & 109.4 \\
\hline $\mathrm{C} 7-\mathrm{C} 6-\mathrm{C} 5$ & $116.01(13)$ & $\mathrm{H} 14 \mathrm{~A}-\mathrm{C} 14-\mathrm{H} 14 \mathrm{~B}$ & 108.0 \\
\hline $\mathrm{C} 7-\mathrm{C} 6-\mathrm{H} 6$ & 122.0 & $\mathrm{C} 8-\mathrm{C} 15-\mathrm{H} 15 \mathrm{~A}$ & 109.5 \\
\hline $\mathrm{C} 5-\mathrm{C} 6-\mathrm{H} 6$ & 122.0 & $\mathrm{C} 8-\mathrm{C} 15-\mathrm{H} 15 \mathrm{~B}$ & 109.5 \\
\hline $\mathrm{C} 6-\mathrm{C} 7-\mathrm{O} 1$ & $125.68(13)$ & $\mathrm{H} 15 \mathrm{~A}-\mathrm{C} 15-\mathrm{H} 15 \mathrm{~B}$ & 109.5 \\
\hline $\mathrm{C} 6-\mathrm{C} 7-\mathrm{C} 2$ & $123.65(13)$ & $\mathrm{C} 8-\mathrm{C} 15-\mathrm{H} 15 \mathrm{C}$ & 109.5 \\
\hline $\mathrm{O} 1-\mathrm{C} 7-\mathrm{C} 2$ & $110.67(12)$ & $\mathrm{H} 15 \mathrm{~A}-\mathrm{C} 15-\mathrm{H} 15 \mathrm{C}$ & 109.5 \\
\hline $\mathrm{C} 1-\mathrm{C} 8-\mathrm{O} 1$ & $110.90(12)$ & $\mathrm{H} 15 \mathrm{~B}-\mathrm{C} 15-\mathrm{H} 15 \mathrm{C}$ & 109.5 \\
\hline $\mathrm{C} 1-\mathrm{C} 8-\mathrm{C} 15$ & $133.45(15)$ & $\mathrm{C} 17-\mathrm{C} 16-\mathrm{C} 21$ & $121.91(13)$ \\
\hline $\mathrm{O} 1-\mathrm{C} 8-\mathrm{C} 15$ & $115.64(13)$ & $\mathrm{C} 17-\mathrm{C} 16-\mathrm{S} 1$ & $119.95(11)$ \\
\hline $\mathrm{C} 4-\mathrm{C} 9-\mathrm{C} 10$ & $111.98(11)$ & $\mathrm{C} 21-\mathrm{C} 16-\mathrm{S} 1$ & $118.02(10)$ \\
\hline $\mathrm{C} 4-\mathrm{C} 9-\mathrm{C} 14$ & $111.49(11)$ & $\mathrm{C} 18-\mathrm{C} 17-\mathrm{C} 16$ & $116.83(13)$ \\
\hline $\mathrm{C} 10-\mathrm{C} 9-\mathrm{C} 14$ & $109.86(12)$ & $\mathrm{C} 18-\mathrm{C} 17-\mathrm{H} 17$ & 121.6 \\
\hline $\mathrm{C} 4-\mathrm{C} 9-\mathrm{H} 9$ & 107.8 & $\mathrm{C} 16-\mathrm{C} 17-\mathrm{H} 17$ & 121.6 \\
\hline $\mathrm{C} 10-\mathrm{C} 9-\mathrm{H} 9$ & 107.8 & $\mathrm{~F} 1-\mathrm{C} 18-\mathrm{C} 19$ & $117.70(14)$ \\
\hline $\mathrm{C} 14-\mathrm{C} 9-\mathrm{H} 9$ & 107.8 & $\mathrm{~F} 1-\mathrm{C} 18-\mathrm{C} 17$ & $118.79(15)$ \\
\hline $\mathrm{C} 11-\mathrm{C} 10-\mathrm{C} 9$ & $111.76(12)$ & $\mathrm{C} 19-\mathrm{C} 18-\mathrm{C} 17$ & $123.50(14)$ \\
\hline $\mathrm{C} 11-\mathrm{C} 10-\mathrm{H} 10 \mathrm{~A}$ & 109.3 & $\mathrm{C} 18-\mathrm{C} 19-\mathrm{C} 20$ & $118.24(14)$ \\
\hline $\mathrm{C} 9-\mathrm{C} 10-\mathrm{H} 10 \mathrm{~A}$ & 109.3 & $\mathrm{C} 18-\mathrm{C} 19-\mathrm{H} 19$ & 120.9 \\
\hline $\mathrm{C} 11-\mathrm{C} 10-\mathrm{H} 10 \mathrm{~B}$ & 109.3 & $\mathrm{C} 20-\mathrm{C} 19-\mathrm{H} 19$ & 120.9 \\
\hline $\mathrm{C} 9-\mathrm{C} 10-\mathrm{H} 10 \mathrm{~B}$ & 109.3 & $\mathrm{C} 19-\mathrm{C} 20-\mathrm{C} 21$ & $120.67(14)$ \\
\hline $\mathrm{H} 10 \mathrm{~A}-\mathrm{C} 10-\mathrm{H} 10 \mathrm{~B}$ & 107.9 & $\mathrm{C} 19-\mathrm{C} 20-\mathrm{H} 20$ & 119.7 \\
\hline $\mathrm{C} 12-\mathrm{C} 11-\mathrm{C} 10$ & $111.91(12)$ & $\mathrm{C} 21-\mathrm{C} 20-\mathrm{H} 20$ & 119.7 \\
\hline $\mathrm{C} 12-\mathrm{C} 11-\mathrm{H} 11 \mathrm{~A}$ & 109.2 & $\mathrm{C} 20-\mathrm{C} 21-\mathrm{C} 16$ & $118.81(14)$ \\
\hline $\mathrm{C} 10-\mathrm{C} 11-\mathrm{H} 11 \mathrm{~A}$ & 109.2 & $\mathrm{C} 20-\mathrm{C} 21-\mathrm{H} 21$ & 120.6 \\
\hline
\end{tabular}




\begin{tabular}{|c|c|c|c|}
\hline $\mathrm{C} 12-\mathrm{C} 11-\mathrm{H} 11 \mathrm{~B}$ & 109.2 & $\mathrm{C} 16-\mathrm{C} 21-\mathrm{H} 21$ & 120.6 \\
\hline $\mathrm{O} 2-\mathrm{S} 1-\mathrm{C} 1-\mathrm{C} 8$ & $-134.55(12)$ & $\mathrm{C} 7-\mathrm{O} 1-\mathrm{C} 8-\mathrm{C} 15$ & $179.79(12)$ \\
\hline $\mathrm{C} 16-\mathrm{S} 1-\mathrm{C} 1-\mathrm{C} 8$ & $114.34(12)$ & $\mathrm{C} 3-\mathrm{C} 4-\mathrm{C} 9-\mathrm{C} 10$ & $-54.46(17)$ \\
\hline $\mathrm{O} 2-\mathrm{S} 1-\mathrm{C} 1-\mathrm{C} 2$ & $42.59(13)$ & $\mathrm{C} 5-\mathrm{C} 4-\mathrm{C} 9-\mathrm{C} 10$ & $126.65(14)$ \\
\hline $\mathrm{C} 16-\mathrm{S} 1-\mathrm{C} 1-\mathrm{C} 2$ & $-68.53(13)$ & $\mathrm{C} 3-\mathrm{C} 4-\mathrm{C} 9-\mathrm{C} 14$ & $69.09(17)$ \\
\hline $\mathrm{C} 8-\mathrm{C} 1-\mathrm{C} 2-\mathrm{C} 7$ & $-0.61(14)$ & $\mathrm{C} 5-\mathrm{C} 4-\mathrm{C} 9-\mathrm{C} 14$ & $-109.80(15)$ \\
\hline $\mathrm{S} 1-\mathrm{C} 1-\mathrm{C} 2-\mathrm{C} 7$ & $-178.13(10)$ & $\mathrm{C} 4-\mathrm{C} 9-\mathrm{C} 10-\mathrm{C} 11$ & $179.30(12)$ \\
\hline $\mathrm{C} 8-\mathrm{C} 1-\mathrm{C} 2-\mathrm{C} 3$ & $179.01(14)$ & $\mathrm{C} 14-\mathrm{C} 9-\mathrm{C} 10-\mathrm{C} 11$ & $54.84(16)$ \\
\hline $\mathrm{S} 1-\mathrm{C} 1-\mathrm{C} 2-\mathrm{C} 3$ & $1.5(2)$ & $\mathrm{C} 9-\mathrm{C} 10-\mathrm{C} 11-\mathrm{C} 12$ & $-54.84(17)$ \\
\hline $\mathrm{C} 7-\mathrm{C} 2-\mathrm{C} 3-\mathrm{C} 4$ & $-0.38(19)$ & $\mathrm{C} 10-\mathrm{C} 11-\mathrm{C} 12-\mathrm{C} 13$ & $54.65(18)$ \\
\hline $\mathrm{C} 1-\mathrm{C} 2-\mathrm{C} 3-\mathrm{C} 4$ & $-179.96(14)$ & $\mathrm{C} 11-\mathrm{C} 12-\mathrm{C} 13-\mathrm{C} 14$ & $-55.75(19)$ \\
\hline $\mathrm{C} 2-\mathrm{C} 3-\mathrm{C} 4-\mathrm{C} 5$ & $-0.09(19)$ & $\mathrm{C} 12-\mathrm{C} 13-\mathrm{C} 14-\mathrm{C} 9$ & $57.1(2)$ \\
\hline $\mathrm{C} 2-\mathrm{C} 3-\mathrm{C} 4-\mathrm{C} 9$ & $-178.99(11)$ & $\mathrm{C} 4-\mathrm{C} 9-\mathrm{C} 14-\mathrm{C} 13$ & $179.29(14)$ \\
\hline $\mathrm{C} 3-\mathrm{C} 4-\mathrm{C} 5-\mathrm{C} 6$ & $0.2(2)$ & $\mathrm{C} 10-\mathrm{C} 9-\mathrm{C} 14-\mathrm{C} 13$ & $-55.97(18)$ \\
\hline $\mathrm{C} 9-\mathrm{C} 4-\mathrm{C} 5-\mathrm{C} 6$ & $179.11(13)$ & $\mathrm{O} 2-\mathrm{S} 1-\mathrm{C} 16-\mathrm{C} 17$ & $-9.39(14)$ \\
\hline $\mathrm{C} 4-\mathrm{C} 5-\mathrm{C} 6-\mathrm{C} 7$ & $0.2(2)$ & $\mathrm{C} 1-\mathrm{S} 1-\mathrm{C} 16-\mathrm{C} 17$ & $102.44(12)$ \\
\hline $\mathrm{C} 5-\mathrm{C} 6-\mathrm{C} 7-\mathrm{O} 1$ & $179.75(13)$ & $\mathrm{O} 2-\mathrm{S} 1-\mathrm{C} 16-\mathrm{C} 21$ & $166.74(12)$ \\
\hline $\mathrm{C} 5-\mathrm{C} 6-\mathrm{C} 7-\mathrm{C} 2$ & $-0.7(2)$ & $\mathrm{C} 1-\mathrm{S} 1-\mathrm{C} 16-\mathrm{C} 21$ & $-81.42(13)$ \\
\hline $\mathrm{C} 8-\mathrm{O} 1-\mathrm{C} 7-\mathrm{C} 6$ & $-179.98(14)$ & $\mathrm{C} 21-\mathrm{C} 16-\mathrm{C} 17-\mathrm{C} 18$ & $2.0(2)$ \\
\hline $\mathrm{C} 8-\mathrm{O} 1-\mathrm{C} 7-\mathrm{C} 2$ & $0.40(15)$ & $\mathrm{S} 1-\mathrm{C} 16-\mathrm{C} 17-\mathrm{C} 18$ & $177.96(11)$ \\
\hline $\mathrm{C} 3-\mathrm{C} 2-\mathrm{C} 7-\mathrm{C} 6$ & $0.8(2)$ & $\mathrm{C} 16-\mathrm{C} 17-\mathrm{C} 18-\mathrm{F} 1$ & $179.49(14)$ \\
\hline $\mathrm{C} 1-\mathrm{C} 2-\mathrm{C} 7-\mathrm{C} 6$ & $-179.50(13)$ & $\mathrm{C} 16-\mathrm{C} 17-\mathrm{C} 18-\mathrm{C} 19$ & $-1.5(2)$ \\
\hline $\mathrm{C} 3-\mathrm{C} 2-\mathrm{C} 7-\mathrm{O} 1$ & $-179.57(11)$ & $\mathrm{F} 1-\mathrm{C} 18-\mathrm{C} 19-\mathrm{C} 20$ & $179.71(16)$ \\
\hline $\mathrm{C} 1-\mathrm{C} 2-\mathrm{C} 7-\mathrm{O} 1$ & $0.13(14)$ & $\mathrm{C} 17-\mathrm{C} 18-\mathrm{C} 19-\mathrm{C} 20$ & $0.7(3)$ \\
\hline $\mathrm{C} 2-\mathrm{C} 1-\mathrm{C} 8-\mathrm{O} 1$ & $0.90(15)$ & $\mathrm{C} 18-\mathrm{C} 19-\mathrm{C} 20-\mathrm{C} 21$ & $-0.3(3)$ \\
\hline $\mathrm{S} 1-\mathrm{C} 1-\mathrm{C} 8-\mathrm{O} 1$ & $178.55(9)$ & $\mathrm{C} 19-\mathrm{C} 20-\mathrm{C} 21-\mathrm{C} 16$ & $0.8(3)$ \\
\hline $\mathrm{C} 2-\mathrm{C} 1-\mathrm{C} 8-\mathrm{C} 15$ & $-179.85(15)$ & $\mathrm{C} 17-\mathrm{C} 16-\mathrm{C} 21-\mathrm{C} 20$ & $-1.7(2)$ \\
\hline $\mathrm{S} 1-\mathrm{C} 1-\mathrm{C} 8-\mathrm{C} 15$ & $-2.2(2)$ & $\mathrm{S} 1-\mathrm{C} 16-\mathrm{C} 21-\mathrm{C} 20$ & $-177.77(13)$ \\
\hline $\mathrm{C} 7-\mathrm{O} 1-\mathrm{C} 8-\mathrm{C} 1$ & $-0.81(15)$ & & \\
\hline
\end{tabular}

Hydrogen-bond geometry $\left(A,{ }^{\circ}\right)$

$\mathrm{Cg} 1$ and $\mathrm{Cg} 2$ are the centroids of the $\mathrm{C} 1 / \mathrm{C} 2 / \mathrm{C} 7 / \mathrm{O} 1 / \mathrm{C} 8$ furan ring and the $\mathrm{C} 2-\mathrm{C} 7$ benzene ring, respectively.

\begin{tabular}{lllll}
\hline$D-\mathrm{H} \cdots A$ & $D-\mathrm{H}$ & $\mathrm{H} \cdots A$ & $D \cdots A$ & $D-\mathrm{H} \cdots A$ \\
\hline $\mathrm{C} 13-\mathrm{H} 13 A \cdots C g 1^{\mathrm{i}}$ & 0.99 & 3.00 & $3.697(1)$ & 128 \\
$\mathrm{C} 14-\mathrm{H} 14 B \cdots C g 2^{\mathrm{i}}$ & 0.99 & 2.91 & $3.569(1)$ & 125 \\
$\mathrm{C} 19-\mathrm{H} 19 \cdots C g 2^{\mathrm{ii}}$ & 0.95 & 2.90 & $3.677(1)$ & 140
\end{tabular}

Symmetry codes: (i) $-x+1,-y,-z+1$; (ii) $x+1, y, z$. 